\title{
Evaluation of the bamboo shoots' development status and nutrition in Sichuan, China
}

\author{
Yuntao Liu ${ }^{1,2, a^{*}}$, Qianqian Tang ${ }^{2}$, Yuxian You², Siqi Zeng ${ }^{2}$, Yiwen $\mathrm{Li}^{2}$, Di Chen², \\ Aiping Liu' ${ }^{2}$, Chaohui Feng ${ }^{2}$, Cheng $\mathrm{Li}^{2}$, Daiwen Chen ${ }^{1}$ \\ ${ }^{1}$ Animal Nutrition Institute, Sichuan Agricultural University, Chengdu 611130, China. \\ ${ }^{2}$ College of Food Science, Sichuan Agricultural University, Yaan 625014, China. \\ $\mathrm{a}^{*}$ lyt_taotao@163.com (corresponding author)
}

Keywords: Bamboo shoots, Processing, Nutrition, Status, Sichuan

\begin{abstract}
As a natural health-care food, bamboo shoots have been quite popular. Mainly distributed in Sichuan and other places in China, they contain not only rich nutrients and vitamins, but also active substances, including various polyphenols, polysaccharides and dietary fibers, with the biological activity of anti-oxidation, anti-free-radicals, blood-sugar-reduction and anti-aging. The processing and utilization of bamboo shoots is extremely popular. Though bamboo shoots have become the polar industry of Sichuan and other places in China, there are still many problems concerning the processing and utilization of bamboo shoots. This paper conducts a clear exposition of China's rich bamboo shoots resources and problems existing in processing and trading of bamboo shoots.
\end{abstract}

\section{Introduction}

China is one of the sources and distribution centers of bamboos. The middle and lower reaches of the Brahmaptura River in the south of the medium and lower reaches of the Yellow River is grown with bamboos. The area south of the Yangtze River boasts the largest number of bamboos. Located in the upper reaches of the Yangtze River, Sichuan belongs to the sub-tropical climate. It has the special basins, high mountains and plateaus. Various kinds of bamboos cover both plains and high attitude areas. It is the mixed bamboo area with the most typical scattering and sympodial bamboos. [1] Now, bamboos in the area have reached 19 categories and more than 160 kinds (There are 73 local bamboo kinds, accounting for $52 \%$ of the total indigenous bamboo kinds). These bamboos cover an area of 700,000 hectares, ranking No.5 nationwide. More than $70 \%$ are sympodial bamboos. The research scale is No.1 in China. Among them, the artificially-made bamboo forests cover an area of 340,000 hectares, accounting for one tenth of the total bamboo area in China. Currently, Sichuan has developed the annual output capability of more than 400,000 tons. $[1,2]$

Sichuan is one of provinces in China with rich bamboo resources. There are many bamboo kinds which can turn out edible bamboo shoots. They are distributed mainly in hills and border mountainous areas of basins. Among them, chimonobambusa qundrangularis, solid bamboos, qiongzhuea tumidinoda, phyllostachys nidularia and fargesia register a high output. chimonobambusa, solid bamboos, qiongzhuea tumidinoda, and fargesia are mainly distributed in Hongya, Xingyang, Muchuan, Emei, Nanchuan, Tianquan, Xuanhan and several counties in border mountainous areas of basins. Phyllostachys pubescens and acanthophyllum pungens are mainly distributed in Changning, Jiang'an, Hejiang, Xingwen and other counties. Other sympodial bamboos, such as chicken-feet bamboos, winter bamboos, flexuose bamboos, bambusa tetilts and Man bamboos, are located in hilly areas of basins. [3]

With the development of economy and the improvement of people's living standards, health has become a focus of the public. As a natural health-care food, bamboo shoots are rich in sugar, protein, dietary fibers, multiple mineral nutrients and vitamins, and important biological activity. Therefore, bamboo shoots have been extremely popular, and even reputed as "Germ of Mountains." Currently, 
bamboo shoots are sold in Asian countries as products. For example, the annual output of bamboo shoots in China has reached about 5 million tons, and its annual output value has exceeded 20 billion RMB, earning foreign exchange of 800 million USD through exports. The per capita consumption of bamboo shoots is also on the increase. From 1995 to 1960, the per capita consumption of bamboo shoots was about $1.2 \mathrm{~kg} /$ person; from 1971 to 1980 , the figure was $2.47 \mathrm{~kg} /$ person; from 1981 to 1991 , the figure increased to $3.08 \mathrm{~kg} /$ person [4-6].

Therefore, to turn the resource advantage of bamboo shoots is of vital importance to the promotion of new countryside construction, the development of bamboo shoots and even the whole bamboo industry.

\section{Safety and Nutritional Value of Bamboo Shoots Resources}

Bamboos are an important part of forest resources, and bamboo shoots are one of the traditional forest vegetables. Forest vegetables are one of the five healthy food types prevailing worldwide. Growing in the forest environment with fresh air, bamboo shoots are free from the contamination of hazardous materials, such as waste gas, wastewater, pesticides, fertilizers and flying dust. Therefore, bamboo shoots have been regarded as the cleanest fresh vegetable, which is extremely healthy to human body. Thus, they have earned the reputation of "Treasure of Forest Sea," "Germ of Mountains" and "Green Food." [7]

Due to rich nutritious value, bamboo shoots are considered as the optimal green food. Its protein is as high as $2.65 \%$. (One of vegetables with the highest protein content) However, their calories are just $104.6 \mathrm{~kJ} / 100 \mathrm{~g}$. The characteristic can contribute to the prevention of cardiovascular diseases, diabetes and other chronic diseases. Moreover, the protein content of bamboo shoots is significantly higher than the average value of the other vegetables, which is $1.45 \%$. (Generally speaking, humans take in food so as to supplement the protein content within body.) Last but not least, bamboo shoots have 17 amino acids required by the human body and the mineral elements, including $\mathrm{Fe}, \mathrm{Mg}, \mathrm{Ca}$ and P. [8]

\section{Major Functions and Activity Ingredients of Bamboo Shoots Resources}

The use of bamboo shoots as a medicine has a time-honored history. Traditional Chinese medicine thinks that bamboo shoots have a sweet flavor and has the function of eliminating phlegm by cooling, relieving internal heat or fever and moistening intestines. Those suffering from heat toxin and pyrophlegm, stomach heat, thirst and constipation, coughing and excessive phlegm, dyspepsia, abdominal fullness and distention can have bamboo shoots to alleviate symptoms of the kind. In Compendium of Materia Medica by LI Shizhen, it is written that "bamboo shoots have the of relieving heat, dissolving phlegm and refreshing stomach."

Bamboo shoots are abundant in active materials, including polyphenols, polysaccharides and dietary fibers, and have the biological activity of anti-oxidation, anti-free-radicals, anti-tumor, blood-sugar-reduction and anti-aging. [9] Park and John studied the anti-oxidant, anti-bacteria and blood-inhibiting angiotension converting enzyme (ACE) activity of polyphenol extractives of black bamboo and bamboo shoots of phyllostachys pubescens. Research results suggest that the polyphenol of bamboo shoots to be tested has prominent anti-oxidant and ACE activity, but showing no anti-bacteria activity. [10] However, in another research program, Park and John asked eight young females aged from 21 to 23 years old to take in $360 \mathrm{~h}$ of bamboo shoots in their daily diet. After the diet continued for six days, their blood fat, blood sugar, excreta, peristalsis and other physical indexes were measured. Results suggested that the intake of bamboo shoots can significantly reduce the total cholesterol in serum, low density lipoprotein cholesterin and atherosis, while excreta and peristalsis frequency were increased significantly, but causing no influence on sugar blood. This indicated that to adopt bamboo shoots as a part of daily diet is beneficial for maintaining a healthy blood lipid level and the digestive tract. [11] Bamboo shoots have an outstanding anti-diabetes activity. Si-Jie Shu and so on employs the Lei bamboo shoots juice to treat diabetes mice induced by alloxan. After a treatment period of 28 days, the Lei bamboo shoots juice 
can significantly reduce the blood sugar, triacylglycerol, NEFA, low density lipoprotein cholesterin and malondialdehyde, and the high density lipoprotein cholesterol, superoxide dismutase and the level of the hepatic glycogen, thus showing effective anti-diabetes function. [12]

\section{Processing and Utilization of Bamboo Shoots Resources}

With China's implementation of the policy of "returning the grain plots to forestry," bamboo kinds which can turn out edible bamboo shoots have been grown by a large area. The rapid development of the bamboo forest industry has also led to the soaring output number of fresh bamboos. At present, bamboo shoots have become an important product and new economic growth point of China's bamboo forest industry. However, due to a short marketing period and small market capacity of fresh bamboo shoots, and the serious backwardness and shortages of fresh bamboo processing and utilization methods and techniques, fresh bamboos are often faced with difficulties of being sold out, low sales prices and are even abandoned by a large amount, thus serious influencing the development of the bamboo shoots agricultural and forestry economy. Obviously, to try to expand fresh bamboo shoots processing and utilization approaches, study and develop new techniques for the comprehensive processing and utilization of fresh bamboo shoots and products, expand he processing and utilization scale of fresh bamboo shoots and product types, improve the added-value and benefits of bamboo shoots processing and utilization are of vital significance to the improvement of the status and development of China's bamboo shoots industry, and even the social and economic benefits of China.

Processing Status of Bamboo Shoots. The processing and production of bamboo shoots is the polar industry of many places in Sichuan. Take Changning County in Yibing City, Sichuan, for example. As a county with a high output of bamboos, Changning has earned the reputation of "China's Bamboo Hometown." There have been about 13 manufacturers engaged in bamboo shoots production and processing. The annual processing output of bamboo shoots is as high as 30,000 tons, and is distributed to all places in China. About $40 \%$ of China's fresh bamboo shoots every year are used for fresh eating and sales; while $60 \%$ are used for further processing. Annually, there is about 120,000 tons of dried bamboo shoots and 240,000 tons of bamboo shoots cans made from fresh bamboo shoots. The bamboo shoots fabricated products include fresh bamboo shoots (boiled bamboo shoots), dried bamboo shoots, salted bamboo shoots, flavored bamboo shoots, quick-freezing bamboo shoots and so on, other by-products of bamboo shoots processing. At present, the bamboo products have achieved seriation. The boiled bamboo shoots have the highest output up to now. Its processing techniques are mature and its production devices are complete. Over the past few years, the flavored bamboo shoots have made great strides. Both flavored bamboo shoots manufacturers and output have accounted for an increasing percentage during the processing of bamboo shoots. The scientific research into flavored bamboo shoots has drawn more and more attention. According flavor characteristics of Sichuan Cuisine, LI Guanghui and ZHONG Shirong processed seasonings of Sichuan Cuisine into bamboo shoots compound seasonings in accordance with the making principle and methods of compound seasonings. [13]

Problems Existing In the Bamboo Processing Industry. Based on viewpoints of relevant literatures, China's bamboo shoots industry has the following problems: 1) low mechanization degree; 2) lack of unified product standards; 3) lack of flagship products; 4) backwardness of mass preservation technology of bamboo shoots; 5) low industrial concentration; 6) lack of product diversity and export market diversity. [5-7, 14]

Development Trend of Bamboo Shoots Processing. Bamboo shoots have become an important raw material of the modern food industry. The development of bamboo foods has promising prospects. The future bamboo shoots processing and production should be more nutritious, healthy and convenient. In order to meet market demands, the bamboo shoots processing industry should achieve breakthroughs in the following aspects: 1) to enhance scientific research, try to develop new products and solve the technical problems of bamboo shoots preservation and processing; 2) to properly distribute the bamboo shoots production bases and choose the proper bamboo kinds for planting; 3) to process semi-finished products or process flavored bamboo shoots; 4) to process 
compound bamboo foods; 5) to develop frozen dried bamboo shoots; 6) to develop medical products and health-care drinks with bamboo shoots juice as the raw material. $[5-7,14]$

\section{Acknowledgements}

This work was supported by Scientific Research Foundation of Sichuan Agricultural University (No. 06021400) and The National Natural Science Foundation of China (No. 31501550) .

\section{References}

[1] T.P. Yi, Bamboos flora of Sichuan, China Forestry Press, Beijing, 1997. (In Chinese)

[2] D.S. Yang, Afforestation techniques of main bamboo species in Sichuan, Sichuan Science and Technology Press, Chengdu, 2005. (In Chinese)

[3] P. Sun, Z. Wang and X.P. Zhang, Bamboo cultivation and management. World Press, Chengdu, 2009. (In Chinese)

[4] F.C. Zhou, Review and prospect of bamboo industry in twenty-first Century, Journal of Bamboo Research. 18 (1999) 1-4. (In Chinese)

[5] Q. Li, G.H. Wang and D.H. Zhang, Processing and trade of bamboo shoot in China, Journal of Zhejiang Forestry Science and Technology. 21 (2001) 38-41. (In Chinese)

[6] W.S. Huang and B.Y. Lu, Advances in deep-proessing technology of bamboo shoots, Scientia Silvae Sinicae. 44 (2008) 118-123. (In Chinese)

[7] G. Chen, Processing and comprehensive utilization of bamboo shoot, Chemical Industry Press, Beijing, 2007. (In Chinese)

[8] J.H. Xiao, Management of bamboo forest in China. Science Press, Beijing, 2010. (In Chinese)

[9] D. Choudhury, J.K. Sahu and G.D. Sharma, Value addition to bamboo shoots:a review, Journal of Food Science and Technology. 49 (2012) 407-414.

[10] E.J. Park and D.Y. John, The antioxidant, angiotensin converting enzyme inhibition activity, and the phenolic compounds of bamboo shoot extracts, LWT-Food Science Technology. 43 (2010) 655-659.

[11] E.J. Park and D.Y. John, Effects of bamboo shoot consumption on lipid profiles and bowel function in healthy young women, Nutrition. 25 (2009) 723-728.

[12] S.J. Shu, H. Shu and Y.S. Lin, Juice of Leizhusun for many plasma factors and hepatic glycogen in rats with diabetes, Chinese Journal of Clinical Rehabilitation. 9 (2005) 232-233. (In Chinese)

[13] G.H. Li and S.R Zhong, Study on processing technology of bamboo shoots with Sichuan cuisine, Sichuan Food and Permentation. 44 (2008) 61-63. (In Chinese)

[14] Q.T. Shi, Present situation and developing prospect of bamboo shoot processing and utilization in China, Journal of Bamboo Research. 22 (2003) 1-4. (In Chinese) 